\title{
Volumetric modulated arc therapy for carotid sparing in the management of early glottic cancer
}

\author{
Young Suk Kim, MD ${ }^{1 *}$, Jaegi Lee, $\mathrm{BS}^{2 *}$, Jong In Park, MS², Wonmo Sung, MS², \\ Sol Min Lee', Gwi Eon Kim, MD, PhD' \\ 'Department of Radiation Oncology, Jeju National University Hospital, Jeju National University School of Medicine, Jeju; \\ ${ }^{2}$ Program in Biomedical Radiation Sciences, Department of Transdisciplinary Studies, Graduate School of Convergence \\ Science and Technology, Seoul National University, Seoul, Korea
}

\begin{abstract}
Purpose: Radiotherapy of the neck is known to cause carotid artery stenosis. We compared the carotid artery dose received between volumetric modulated arc therapy (VMAT) and conventional fixed-field intensity-modulated radiotherapy (IMRT) plans in patients with early glottic cancer.

Materials and Methods: Twenty-one early glottic cancer patients who previously underwent definitive radiotherapy were selected for this study. For each patient, double arc VMAT, 8-field IMRT, 3-dimensional conformal radiotherapy (3DCRT), and lateral parallel-opposed photon field radiotherapy (LPRT) plans were created. The 3DCRT plan was generated using lateral parallel-opposed photon fields plus an anterior photon field. VMAT and IMRT treatment plan optimization was performed under standardized conditions to obtain adequate target volume coverage and spare the carotid artery. Dose-volume specifications for the VMAT, IMRT, 3DCRT, and LPRT plans were calculated with radiotherapy planning system. Monitor units (MUs) and delivery time were measured to evaluate treatment efficiency.

Results: Target volume coverage and homogeneity results were comparable between VMAT and IMRT; however, VMAT was superior to IMRT for carotid artery dose sparing. The mean dose to the carotid arteries in double arc VMAT was reduced by 6.8\% compared to fixed-field IMRT ( $p<0.001)$. The MUs for VMAT and IMRT were not significantly different $(p=0.089)$. VMAT allowed an approximately two-fold reduction in treatment delivery time in comparison to IMRT ( 3 to 5 minutes vs. 5 to 10 minutes).

Conclusion: VMAT resulted in a lower carotid artery dose compared to conventional fixed-field IMRT, and maintained good target coverage in patients with early glottic cancer.
\end{abstract}

Keywords: Volumetric modulated arc therapy, Intensity-modulated radiotherapy, Glottis, Cancer, Carotid arteries

\section{Introduction}

Traditionally, early glottic cancer (T1-2 NO), treated with local radiotherapy alone has shown 5 -year local control rates of $80 \%-90 \%$ [1]. Lateral parallel-opposed photon field radiotherapy (LPRT) has been used to treat this type of cancer [2]. However, because the carotid arteries are located close to the target region, they often receive a full dose of radiation in LPRT [3]. Several publications have reported on radiationinduced carotid artery atherosclerosis $[4,5]$, stenosis [6],

Received 10 September 2015, Revised 29 October 2015, Accepted 17 November 2015.

Correspondence: Gwi Eon Kim, MD, PhD, Department of Radiation Oncology, Jeju National University Hospital, Jeju National University School of Medicine, 15 Aran 13-gil, Jeju 63241, Korea. Tel: +82-64-717-1330, Fax: +82-64-717-1169,

E-mail: gekim@yuhs.ac

*Young Suk Kim and Jaegi Lee are contributed for this work equally.

(c) This is an Open Access article distributed under the terms of the Creative Commons Attribution Non-Commercial License (http://creativecommons.org/ licenses/by-nc/4.0/) which permits unrestricted non-commercial use, distribution, and reproduction in any medium, provided the original work is properly cited.

www.e-roj.org 
and consecutive ischemic strokes [7] as a complication of radiotherapy.

Recently, carotid sparing conventional fixed-field intensitymodulated radiotherapy (IMRT) and volumetric modulated arc therapy (VMAT) have been reported by several investigators, mainly as comparative studies based on small samples [2,815]. Prior treatment planning studies comparing VMAT and conventional IMRT showed that VMAT had equivocal to superior dosimetric outcomes with respect to carotid sparing $[9,11-13]$. In this study, we performed a dosimetric comparison between conventional fixed-field IMRT and VMAT to assess whether the latter was more beneficial for carotid artery sparing in the treatment of early glottic cancer. For VMAT and IMRT plan optimization, the same dose constraints were used to minimize any variation introduced by the treatment planners.

\section{Materials and Methods}

\section{Patient selection}

The medical records of patients with early glottic cancer who underwent definitive radiotherapy between August 2009 and February 2015 at Jeju National University Hospital were retrospectively reviewed. Our hospital Institutional Review Board approved this study. Twenty-one early glottic squamous cell carcinoma patients, Tis NO $(n=2)$, T1 NO $(n=14)$, and T2 $\mathrm{NO}(\mathrm{n}=5)$, were selected for this study (all male). Exclusion criteria included postoperative radiotherapy $(n=1)$, T3 or lymph node positivity ( $n=2)$, or T2 with vocal cord fixation ( $n=0)$. One patient (T2 No) underwent planning computed tomography (CT) simulation, but he refused further treatment due to old age. The clinical stage was determined according to the American Joint Committee on Cancer staging system, 7th edition. Patients were staged using direct laryngoscopy and CT $[9,16]$.

\section{Planning CT simulation}

The patient was immobilized in a supine position with a short thermoplastic mask. The planning CT scans were performed using a 16-slice CT scanner (Brilliance CT Big Bore; Philips Medical Systems, Cleveland, $\mathrm{OH}, \mathrm{USA})$ with a $0.1 \mathrm{~cm}(\mathrm{n}=1), 0.2$ $\mathrm{cm}(\mathrm{n}=1), 0.3 \mathrm{~cm}(\mathrm{n}=17)$, or $0.5 \mathrm{~cm}(\mathrm{n}=2)$ slice thickness. Intravenous contrast media was used in 15 patients. The CT data were then transferred to a treatment planning system (Eclipse ver. 8.6; Varian Medical Systems Inc., Palo Alto, CA, USA). Treatment was conducted using a linear accelerator with the Millennium 120-leaf multi-leaf collimators (MLCs) system
(Clinac iX; Varian Medical Systems Inc.). Patient set-up was verified weekly by $\mathrm{kV}$ portal images using the On-Board Imager system (Varian Medical Systems Inc.) prior to treatment [8].

\section{The clinical target volume and planning target volume contouring}

The clinical target volume (CTV) included the false and true vocal cords, the anterior and posterior commissures, the arytenoids, and the subglottic region, extending from the superior thyroid notch to the bottom of the cricoid cartilage [8] Laterally, the CTV was limited by the thyroid cartilage [10]. The posterior border included the anterior margin of the vertebral bodies. The superior border was higher for T2 tumors with supraglottic extension (up to the hyoid bone inferior margin). The inferior border was lower for T2 tumors with subglottic extension $(0.3 \mathrm{~cm}$ inferior to the cricoid cartilage inferior margin). The superior-inferior field size ranged from $5 \mathrm{~cm}$ to 6 $\mathrm{cm}$. The anterior border had an approximately $1 \mathrm{~cm}$ falloff in the LPRT treatment plans [17]. The CTVs were all contoured by one radiation oncologist. A planning target volume (PTV) was generated by expanding the CTV by $0.3 \mathrm{~cm}$ in all directions. Treatment was directed to the larynx without elective nodal irradiation. Organs at risk (OARs), including the thyroid gland, bilateral carotid arteries, and spinal cord, were delineated. The combined right and left carotid artery OAR volumes were considered the carotid artery OAR volume. The carotid arteries and spinal cord volumes were extended $1.5 \mathrm{~cm}$ cranially and caudally to the PTV [9].

\section{Treatment planning}

For each patient, VMAT, IMRT, 3-dimensional conformal radiotherapy (3DCRT) using three ports (lateral parallelopposed photon fields plus the anterior photon field), and LPRT plans were created by one treatment planner.

A combination of $30^{\circ}$ and $45^{\circ}$ wedges was used to modify the lateral beam in the LPRT and 3DCRT plans. The lateral fields were equally weighted. The anterior field was optimally weighted to provide adequate PTV coverage in the 3DCRT plans. The block margin was set uniformly $1 \mathrm{~cm}$ around the PTV for both the lateral and anterior fields in the LPRT and 3DCRT plans.

VMAT treatment plans utilized a double arc therapy approach. Each arcs of the VMAT plans used a $358^{\circ}$ rotation from $179^{\circ}$ to $181^{\circ}$ for counter-clockwise (CCW) gantry rotation and from $181^{\circ}$ to $179^{\circ}$ for clockwise (CW) gantry rotation. The collimator angles for CCW and CW gantry rotation were $15^{\circ}$ and $345^{\circ}$, respectively. Eight beam angles of $160^{\circ}, 100^{\circ}$, 
$60^{\circ}, 40^{\circ}, 320^{\circ}, 300^{\circ}, 260^{\circ}$, and $200^{\circ}$ were preselected for IMRT centered on the PTV, and the collimator angles for all IMRT beam angles were $5^{\circ}$.

For VMAT and IMRT plan optimization, the same dose constraints were used for comparison (Table 1). Plans were optimized without $0.3 \mathrm{~cm}$ of skin surface to avoid forcing the dose to a low density area of the PTV. A shell structure was generated outside the PTV from $1.5 \mathrm{~cm}$ to $0.3 \mathrm{~cm}$ at the border of the PTV, which was taken into account during optimization. In addition, planning organ at risk volumes (PRVs) were created for the spinal cord and both carotid arteries with 0.5 $\mathrm{cm}$ of outer margin from each organ. The PRVs of the thyroid gland and both carotid arteries were subtracted by the $0.3 \mathrm{~cm}$ margin of the PTV for a smooth dose gradient for areas that overlapped between the PTV and the OARs (the thyroid gland and both carotid arteries).

A bolus was not required for these plans. All plans were generated with a $6 \mathrm{MV}$ photon beam that utilized MLCs for blocking [9].

All plans were created with a prescription dose per fraction of $2.25 \mathrm{~Gy}$ to a total dose of $65.25 \mathrm{~Gy}$. Plans were normalized so that $\geq 95 \%$ of the PTV received $95 \%$ of the prescription dose (not 100\% because of low-density areas in the PTV) [9].

\section{Plan analysis}

Dose-volume data for the 21 patients were collected from the Eclipse treatment planning system. Specific dosimetric values of interest in this analysis included $D_{\text {mean }}, D_{51}$ and $D_{95}$ for PTV; $D_{\text {mean }}, D_{\text {minı }}, D_{\text {max }}, V_{35 G y}$ and $V_{50 G y}$ for the carotid arteries; $D_{\text {meanı }}$ $D_{\min }$ and $D_{\max }$ for the thyroid gland; and $D_{\max }$ for the spinal cord. $D_{\text {mean }}$ represented the average dose (total dose divided by volume) to each structure. $D_{\min }$ and $D_{\max }$ represented the minimum and maximum measurable doses to each structure, respectively. $D_{5}$ and $D_{95}$ represented the dose received by at least $5 \%$ and $95 \%$ of the PTV, respectively. $V_{35 G y}$ and $V_{50 G y}$ represented the volume irradiated by at least $35 \mathrm{~Gy}$ and $50 \mathrm{~Gy}$ to the carotid arteries. $V_{35 \mathrm{~Gy}}$ and $\mathrm{V}_{50 \mathrm{~Gy}}$ of the carotid arteries were analyzed because the dose-response threshold for intimal-medial thickness of the carotid arteries was only statistically significant at a dose of $\geq 35-50$ Gy [6]. The homogeneity index $(H I)$ was defined as $\left(D_{5}-D_{95}\right) / D_{\text {mean }}[18]$ We evaluated PTV coverage by calculating HI. Monitor units (MUs) and delivery time were measured to evaluate treatment efficiency.

\section{Statistical analysis}

Statistical analysis was performed using IBM SPSS ver. 22.0 (IBM, Armonk, NY, USA). A p-value less than 0.05 was considered statistically significant. A paired t-test was used to calculate the difference within each planning technique after determining normality using the Shapiro-Wilk test. Otherwise, the Wilcoxon signed-rank test was used when normality could not be assumed.

\section{Results}

Four treatment plans were evaluated for each of the 21 patients. In Table 2, the numerical findings from the dosevolume histogram analysis of the PTV and main OARs are reported. Data are presented as averages across the patients and errors indicate inter-patient variability at one standard deviation level.

PTV coverage for the four treatment planning techniques was comparable except for 3DCRT (Table 2). The PTV HI was

Table 1. The dose constraint for IMRT and VMAT

\begin{tabular}{lccc}
\multicolumn{1}{c}{ Structure } & Volume (\%) & Dose (Gy) & Relative weight \\
\hline PTV & 0 & 66.30 & 70 \\
& 2 & 66.20 & 70 \\
& 98 & 66.00 & 70 \\
Shell & 100 & 65.90 & 70 \\
PRV of the carotid arteries - (PTV + 0.3 cm) & 0 & 59.99 & 100 \\
& 20 & 35.00 & 50 \\
Thyroid gland - (PTV + 0.3 cm) & 40 & 25.00 & 50 \\
PRV of the spinal cord & 20 & 25.00 & 50 \\
\end{tabular}

IMRT, intensity-modulated radiotherapy; VMAT, volumetric modulated arc therapy; PTV, planning target volume; PRV, planning organ at risk volume.

${ }^{\text {a) }}$ A $0.3-\mathrm{cm}$ isotropic margin of the PTV was subtracted from the PRV of the carotid arteries. ${ }^{\text {b) }} \mathrm{A} 0.3-\mathrm{cm}$ isotropic margin of the PTV was subtracted from the thyroid gland. 
Table 2. Comparison of average dosimetric values by planning technique $(n=21)$

\begin{tabular}{|c|c|c|c|c|}
\hline Index & VMAT & IMRT & 3DCRT & LPRT \\
\hline \multicolumn{5}{|l|}{ PTV } \\
\hline Mean dose (Gy) & $64.8 \pm 0.8$ & $65.0 \pm 0.8$ & $65.9 \pm 1.7$ & $65.1 \pm 1.2$ \\
\hline $\mathrm{D}_{5}(\mathrm{~Gy})$ & $66.8 \pm 1.2$ & $66.8 \pm 1.1$ & $68.6 \pm 2.1$ & $67.1 \pm 1.9$ \\
\hline$D_{95}(G y)$ & $62.0 \pm 0.0$ & $62.0 \pm 0.0$ & $62.0 \pm 0.0$ & $62.0 \pm 0.0$ \\
\hline Homogeneity index ${ }^{a)}$ & $0.07 \pm 0.02$ & $0.07 \pm 0.02$ & $0.10 \pm 0.03$ & $0.08 \pm 0.03$ \\
\hline \multicolumn{5}{|l|}{ Carotid arteries } \\
\hline Mean dose (Gy) & $22.2 \pm 1.9$ & $23.9 \pm 1.9$ & $49.0 \pm 5.2$ & $54.4 \pm 4.3$ \\
\hline Minimum dose (Gy) & $2.0 \pm 0.6$ & $1.5 \pm 0.2$ & $3.3 \pm 1.0$ & $3.4 \pm 1.2$ \\
\hline Maximum dose (Gy) & $55.9 \pm 6.4$ & $57.1 \pm 6.4$ & $66.4 \pm 2.3$ & $66.6 \pm 1.9$ \\
\hline \multirow{2}{*}{$V_{35 G y}\left(\mathrm{~cm}^{3}\right)$} & $1.2 \pm 0.5$ & $1.6 \pm 0.2$ & $5.1 \pm 1.3$ & $5.4 \pm 1.4$ \\
\hline & $18.2 \pm 6.5^{b)}$ & $24.2 \pm 8.6^{b)}$ & $80.3 \pm 6.8^{b)}$ & $83.7 \pm 6.6^{b)}$ \\
\hline \multirow{2}{*}{$V_{50 G y}\left(\mathrm{~cm}^{3}\right)$} & $0.1 \pm 0.1$ & $0.2 \pm 0.2$ & $4.0 \pm 1.2$ & $5.0 \pm 1.3$ \\
\hline & $1.5 \pm 1.9^{b)}$ & $2.9 \pm 2.7^{b)}$ & $62.6 \pm 13.4^{b)}$ & $78.2 \pm 7.1^{b)}$ \\
\hline Thyroid gland mean dose (Gy) & $20.0 \pm 5.8$ & $20.1 \pm 5.4$ & $41.3 \pm 9.4$ & $41.5 \pm 9.7$ \\
\hline Spinal cord maximum dose (Gy) & $36.7 \pm 1.6$ & $36.3 \pm 3.1$ & $22.3 \pm 1.5$ & $3.7 \pm 1.4$ \\
\hline
\end{tabular}

VMAT, volumetric modulated arc therapy; IMRT, intensity-modulated radiotherapy; 3DCRT, 3-dimensional conformal radiotherapy; LPRT, lateral parallel-opposed photon field radiotherapy; PTV, planning target volume; $D_{5}$, dose received by at least 5\% volume of the planning target volume; $D_{95}$ dose received by at least $95 \%$ volume of the planning target volume; $D_{\text {mean }}$ the mean dose to the planning target volume; $V_{35 G y}$ volume irradiated at least $35 \mathrm{~Gy}$ on the carotid arteries; $V_{50 G y}$ volume irradiated at least $50 \mathrm{~Gy}$ on the carotid arteries.

${ }^{a}$ Homogeneity index $=\left(D_{5}-D_{95}\right) / D_{\text {mean. }}{ }^{\text {b) }}$ The values are presented as percent volume of the parameter.

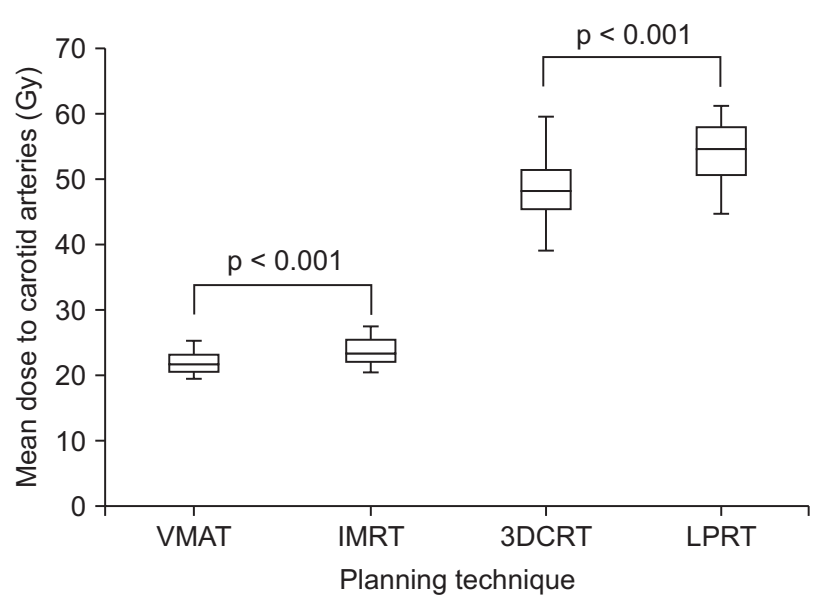

Fig. 1. Box and Whisker plot of the mean dose to both carotid arteries for each treatment planning technique. The horizontal line indicates the median, the box covers the 25\%ile to 75\%ile, and the upper and lower bars are the maximum and minimum values, respectively. VMAT, volumetric modulated arc therapy; IMRT, intensity-modulated radiotherapy; 3DCRT, 3-dimensional conformal radiotherapy; LPRT, lateral parallel-opposed photon field radiotherapy.

significantly worse in the 3DCRT plan than in the other three techniques $(p<0.001)$ because the anterior radiation field without beam modulation could not cover the target area effectively. In two cases especially, the upper level of the PTV encompassed the lower level of the chin. The upper and posterior parts of the PTV could not be covered by the anterior radiation field due to photon beam attenuation in these cases. In addition, using the anterior field, the oblique skin surface of the glottis larynx influenced hot spots at the superior level of the PTV. For these reasons, the target coverage of the 3DCRT plan was highly dependent on the patient's geometry. Half of the 3DCRT plans allowed similar PTV homogeneity with fewer doses to the carotid arteries than LPRT plans. Anterior commissure coverage for the LPRT, IMRT, and VMAT plans was comparable, where $\geq 95 \%$ of the PTV received $95 \%$ of the prescription dose.

The average mean doses to the carotid arteries were 54.4 Gy (range, 45.0 to $61.4 \mathrm{~Gy}$ ), $49.0 \mathrm{~Gy}$ (range, 39.4 to $59.8 \mathrm{~Gy}$ ), $23.9 \mathrm{~Gy}$ (range, 20.8 to $27.5 \mathrm{~Gy}$ ), and $22.2 \mathrm{~Gy}$ (range, 19.4 to $25.6 \mathrm{~Gy}$ ) for LPRT, 3DCRT, IMRT, and VMAT, respectively (Fig. 1). Compared to LPRT, all other treatment modalities showed superiority for dose sparing of the carotid arteries ( $p<0.001$ ). The mean dose to the carotid arteries for VMAT was significantly lower compared to IMRT ( $p<0.001)$. $V_{35 G y}$ and $V_{50 G y}$ of the carotid arteries for VMAT were significantly lower compared to IMRT ( $p<0.001)$. Dose distributions for a representative case are shown in Fig. 2. A dose-volume histogram of a representative case is shown in Fig. 3.

Compared with LPRT, IMRT and VMAT delivered a noticeably lower mean dose to the thyroid gland ( $<$ 0.001) (Table 2). We noted that the average mean dose to the thyroid gland was 

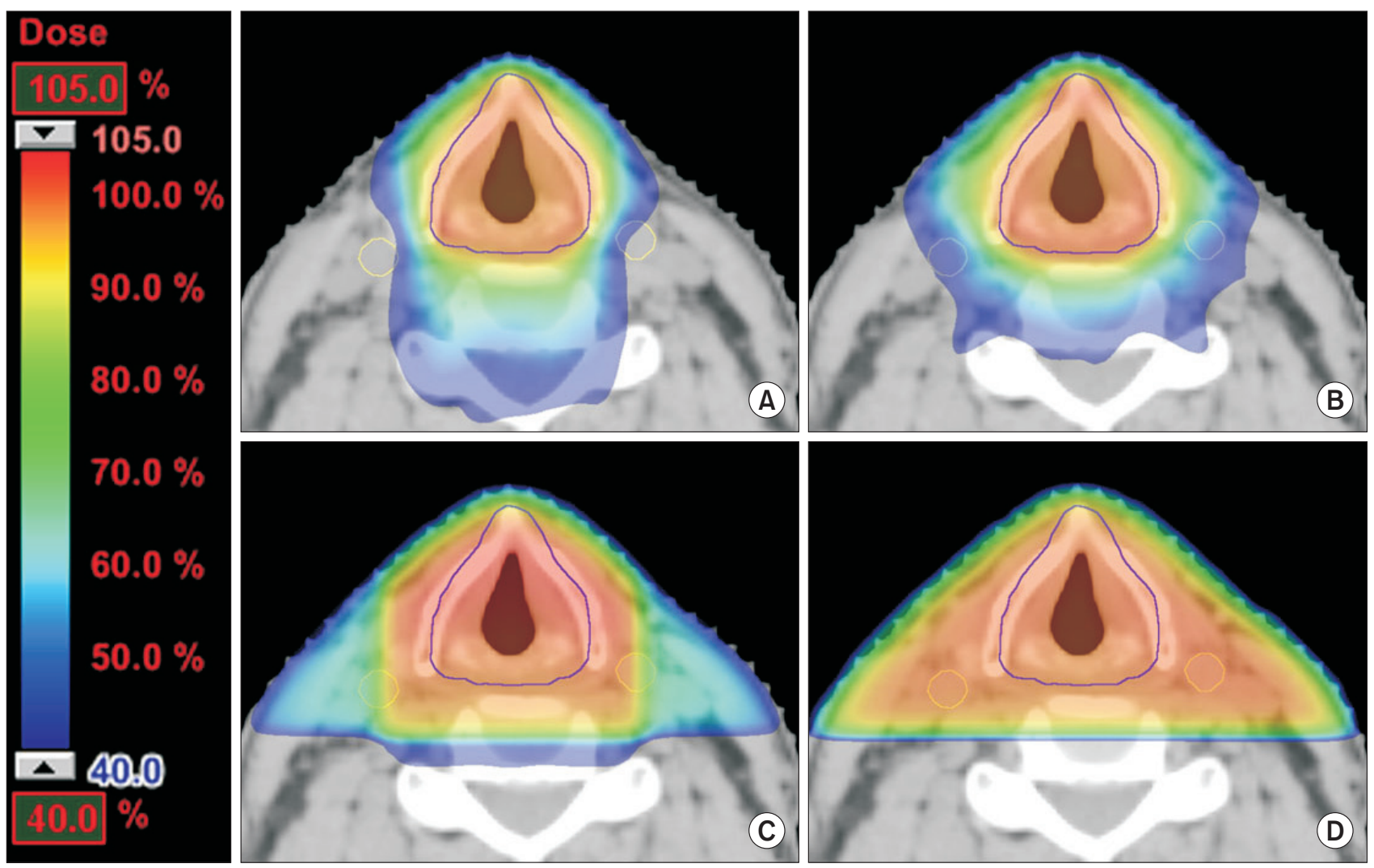

Fig. 2. Isodose curves on an axial slice for a representative case planned with (A) volumetric modulated arc therapy, (B) intensitymodulated radiotherapy, (C) 3-dimensional conformal radiotherapy, and (D) lateral parallel-opposed photon field radiotherapy. The carotid arteries were delineated in yellow line.

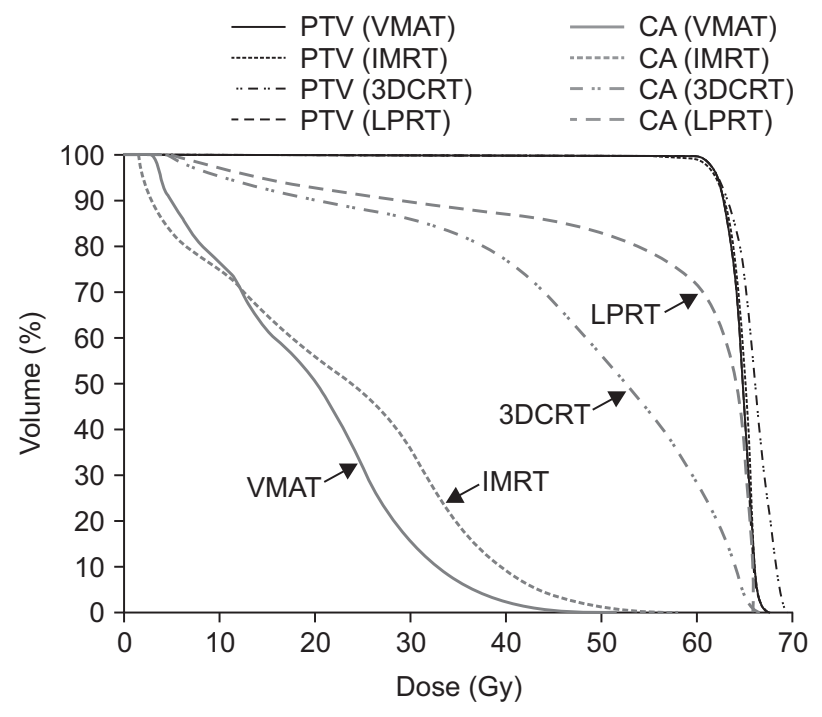

Fig. 3. Dose-volume histogram of the planning target volume (PTV) and the carotid arteries (CA) for the four treatment modalities in a representative case. VMAT, volumetric modulated arc therapy; IMRT, intensity-modulated radiotherapy; 3DCRT, 3-dimensional conformal radiotherapy; LPRT, lateral parallelopposed photon field radiotherapy. similar between IMRT and VMAT ( $p=0.801)$. The difference between LPRT and 3DCRT also was not statistically significant ( $p$ $=0.432$ ). For the spinal cord, the average maximum doses were greatly increased in 3DCT, IMRT, and VMAT (22.3 Gy, $36.3 \mathrm{~Gy}$, and $36.7 \mathrm{~Gy}$, respectively) compared to LPRT (3.7 Gy). However, the maximum doses to the spinal cord did not exceed $45 \mathrm{~Gy}$ for the four treatment plans (Table 2).

The patient-averaged MUs per fraction of 2.25 Gy were 657, 632, 260, and 273 for VMAT, IMRT, 3DCRT, and LPRT, respectively. The MUs for VMAT and IMRT were not significantly different ( $p=0.089)$. For VMAT, each individual arc could be delivered in 75 seconds $\left(4.8^{\circ} / \mathrm{s}\right)$ without heavy MLCs modulation. With the reposition of the collimator and MLCs, most of the double arc VMAT plans could be delivered within 3 to 5 minutes. The overall treatment time for fixed-field IMRT was between 5 and 10 minutes.

\section{Discussion and Conclusion}

Given the relatively limited literature on the application 
of VMAT for early glottic cancer $[2,8]$, there have been few publications on qualitative comparison between VMAT and IMRT [9,11-13]. Matthiesen et al. [9] reported a dosimetric comparison between IMRT and VMAT for the treatment of early glottic cancer. In their study, each course of treatment was tailored to each patient in order to obtain the 'best achievable plan' considering PTV coverage and OAR volume dose [9]. In contrast to these studies, to our knowledge our work is the first to use the same dose constraints during optimization and normalization of VMAT and IMRT for early glottic cancer (Table 1). We compared differences in doses of radiation to the carotid arteries between four treatment modalities under standardized conditions, with a focus on the comparison between VMAT and IMRT.

The main highlight of this study was that VMAT was superior for carotid artery sparing compared to IMRT. The mean doses to the carotid arteries in double arc VMAT were reduced by $6.8 \%$ compared to fixed-field IMRT ( $p<0.001)$. One possible reason is that the carotid arteries were included by the beam path of preselected IMRT fields. With the same dose constraints, VMAT could prevent irradiation of the carotid arteries using 177 control points per each full arc during optimization. The normal tissue complication probabilities calculation for the carotid arteries was not performed due to the unavailability of parameters in the literature [9].

One of the advantages of the VMAT technique found in this study was the significant reduction in the treatment delivery time per fraction [19]. No additional gantry moving time is required for VMAT because the area is continuously exposed to radiation during gantry motion. Conventional fixed-field IMRT included extra time because of the need to reposition the gantry and to reprogram the linear accelerator after every field $[19,20]$. Rosenthal et al. [21] mentioned that IMRT treatment times were similar to LPRT using a virtual edge. In our study, LPRT and 3DCRT should have been faster than IMRT because each plan selected three-field IMRT $\left(0^{\circ}, 70^{\circ}\right.$, and $\left.290^{\circ}\right)$ in their study while eight-field IMRT was used in this trial. Our study expands on what is known about VMAT from the comparison of IMRT with LPRT. Treatment time for single arc VMAT should be similar to LPRT or 3DCRT because the beam-on time is the same as the gantry motion and treatment time. Double arc VMAT requires 1 to 2 minutes more than single arc VMAT. The fast treatment time reduces intra-fraction motion caused by swallowing and body twisting with the loss of concentration on the couch [22].

Irradiation-induced inflammatory reaction in the carotid artery wall triggers a series of events involving the endothelial cells, smooth muscle cells, cytokines, and growth factors that result in changes in the carotid artery wall [23]. Electron microscopy demonstrates swelling and detachment of endothelial cells, splitting of the basement membranes, and subintimal foam cells [24]. Atrophy of the smooth muscle cells of the media leads to replacement hyaline and fibrinoid changes in the carotid artery [25]. Rotman et al. [26] reported marked atherosclerotic changes with calcification of the common carotid artery in the field of irradiation.

In this study, we evaluated only double arc VMAT plans for target coverage and sparing of OARs. Double arc VMAT is better than single arc for target coverage and sparing of OARs in most cases, so we decided to evaluate double arc VMAT plans [27]. It is debatable whether single or multiple arcs should be applied to realize proper volumetric modulated arc techniques [27]. For head and neck cancer, double arc VMAT plans showed fewer doses to the parotid glands than single arc VMAT plans with better target coverage [28]. Target motion is a greater concern with VMAT and IMRT than with conventional radiotherapy techniques because of the longer treatment times [10]. Single arc VMAT would be one of the options for reducing delivery time while maintaining proper target coverage.

In our study, one patient, a 76-year-old male, had an ischemic stroke in the middle cerebral artery region before diagnosis of early glottic cancer. Doppler ultrasound evaluation revealed right proximal internal carotid artery stenosis of a moderate degree (about 50\% to 69\%). One year later, he was diagnosed with early glottic cancer (T1 NO), and was treated with definitive radiotherapy using LPRT. He underwent follow-up Doppler ultrasound at 1 year and 9 months after radiotherapy. There was no significant interval change in right proximal internal carotid artery stenosis. We think that the follow-up period was too short to reveal an interval increase in the carotid artery stenosis in this patient $[6,7]$. Carotid artery sparing using VMAT may be beneficial for reducing the risk of future stroke in cases with ischemic stroke history.

We have treated one patient with this VMAT technique, and another patient with this IMRT technique, respectively. Based on these data, we are planning a prospective study to evaluate the carotid artery stenosis between LPRT and VMAT using Doppler ultrasound [7].

A limitation of our study was the spinal cord doses in VMAT and IMRT. There are two studies focused on IMRT with the anterior fields of the gantry angle [11,13], which could limit the dose to the spinal cord to less than 15 Gy. On the contrary, a $360^{\circ}$ angle was used for VMAT and IMRT in our study because full $360^{\circ}$ gantry angles resulted in better sparing of the carotid 
artery than the plans using only the anterior fields [2]. The maximum doses to the spinal cord in VMAT and IMRT were significantly higher than LPRT and 3DCRT $(p<0.001)$, although the doses to the spinal cord were within tolerance. The other limitation was the dose constraint during optimization. In real clinical situations, the most achievable plan is the best option for treatment. In our study, minimized dose constraints were biased to compare VMAT with IMRT. These optimization strategies were selected to modulate MLCs similarly for all 21 patients. It was possible to reduce the doses to OARs using tighter dose constraints than those we performed, which depended on the geometry of the patients, like the volume and position of each OAR. Finding the best optimization strategy for each patient was not within the scope of our study. Another potential limitation was the lack of CTV contouring consensus for IMRT, although anatomical landmarks are well known for LPRT. Inter-observer variation on CTV contouring may affect dosimetric results.

VMAT was compared to IMRT for treatment of early glottic cancer. Although excellent dosimetric results were achieved with IMRT, VMAT allowed better sparing of the carotid arteries, with uncompromised target coverage. These dosimetric advantages could be beneficial for patients with a history of ischemic stroke history and moderate atherosclerotic changes in the carotid artery.

\section{Conflict of Interest}

No potential conflict of interest relevant to this article was reported.

\section{Acknowledgments}

This work was supported by a research grant from the Jeju National University Hospital research fund of Jeju National University in 2013.

\section{References}

1. Mendenhall WM, Werning JW, Hinerman RW, Amdur RJ, Villaret DB. Management of T1-T2 glottic carcinomas. Cancer 2004;100:1786-92.

2. Riegel AC, Antone J, Schwartz DL. Comparative dosimetry of volumetric modulated arc therapy and limited-angle static intensity-modulated radiation therapy for early-stage larynx cancer. Med Dosim 2013;38:66-9.

3. Janssen S, Glanzmann C, Huber G, Studer G. Risk-adapted partial larynx and/or carotid artery sparing modulated radiation therapy of glottic cancer. Radiat Oncol 2014;9:136.

4. Cheng SW, Ting AC, Wu LL. Ultrasonic analysis of plaque characteristics and intimal-medial thickness in radiationinduced atherosclerotic carotid arteries. Eur J Vasc Endovasc Surg 2002;24:499-504.

5. Kim JH, Jenrow KA, Brown SL. Mechanisms of radiationinduced normal tissue toxicity and implications for future clinical trials. Radiat Oncol J 2014;32:103-15.

6. Martin JD, Buckley AR, Graeb D, Walman B, Salvian A, Hay JH. Carotid artery stenosis in asymptomatic patients who have received unilateral head-and-neck irradiation. Int J Radiat Oncol Biol Phys 2005;63:1197-205.

7. Wilbers J, Dorresteijn LD, Haast $R$, et al. Progression of carotid intima media thickness after radiotherapy: a long-term prospective cohort study. Radiother Oncol 2014;113:359-63.

8. Kim ES, Yeo SG. Volumetric modulated arc radiotherapy sparing the thyroid gland for early-stage glottic cancer: a dosimetrical analysis. Oncol Lett 2014;7:1987-91.

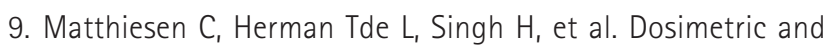
radiobiologic comparison of $3 \mathrm{D}$ conformal, IMRT, VMAT and proton therapy for the treatment of early-stage glottic cancer. J Med Imaging Radiat Oncol 2015;59:221-8.

10. Chera BS, Amdur RJ, Morris CG, Mendenhall WM. Carotidsparing intensity-modulated radiotherapy for early-stage squamous cell carcinoma of the true vocal cord. Int J Radiat Oncol Biol Phys 2010;77:1380-5.

11. Camingue $P$, Christian R, Ng D, Williams P, Amin M, Roniger DL. Comparison of external beam treatment techniques for T1-2, NO, MO glottic cancers. Med Dosim 2012;37:221-4.

12. Atalar B, Gungor G, Caglar H, Aydin G, Yapici B, Ozyar E. Use of volumetric modulated arc radiotherapy in patients with early stage glottic cancer. Tumori 2012;98:331-6.

13. Gomez D, Cahlon O, Mechalakos J, Lee N. An investigation of intensity-modulated radiation therapy versus conventional two-dimensional and 3D-conformal radiation therapy for early stage larynx cancer. Radiat Oncol 2010;5:74.

14. Osman SO, Astreinidou E, de Boer HC, et al. IMRT for imageguided single vocal cord irradiation. Int J Radiat Oncol Biol Phys 2012;82:989-97.

15. Osman SO, Astreinidou E, Levendag PC, Heijmen BJ. Impact of geometric variations on delivered dose in highly focused single vocal cord IMRT. Acta Oncol 2014;53:278-85.

16. Kim TH, Kim MS, Choi SH, et al. Postoperative radiotherapy in salivary ductal carcinoma: a single institution experience. Radiat Oncol J 2014;32:125-31.

17. Ang KK, Garden AS. Radiotherapy for head and neck cancers: indications and techniques. 3rd ed. Philadelphia, PA: Lippincott Williams \& Wilkins; 2006.

18. Zhang $P$, Happersett $L$, Hunt $M$, Jackson $A$, Zelefsky $M$, 
Mageras G. Volumetric modulated arc therapy: planning and evaluation for prostate cancer cases. Int J Radiat Oncol Biol Phys 2010;76:1456-62.

19. White $P$, Chan KC, Cheng KW, Chan KY, Chau MC. Volumetric intensity-modulated arc therapy vs conventional intensitymodulated radiation therapy in nasopharyngeal carcinoma: a dosimetric study. J Radiat Res 2013;54:532-45.

20. Scorsetti M, Bignardi M, Clivio A, et al. Volumetric modulation arc radiotherapy compared with static gantry intensitymodulated radiotherapy for malignant pleural mesothelioma tumor: a feasibility study. Int J Radiat Oncol Biol Phys 2010;77: 942-9.

21. Rosenthal DI, Fuller CD, Barker JL Jr, et al. Simple carotidsparing intensity-modulated radiotherapy technique and preliminary experience for T1-2 glottic cancer. Int J Radiat Oncol Biol Phys 2010;77:455-61.

22. Clivio A, Fogliata A, Franzetti-Pellanda $A$, et al. Volumetricmodulated arc radiotherapy for carcinomas of the anal canal: a treatment planning comparison with fixed field IMRT. Radiother Oncol 2009;92:118-24.
23. Abayomi OK. Neck irradiation, carotid injury and its consequences. Oral Oncol 2004;40:872-8.

24. Ackerman LV. The pathology of radiation effect of normal and neoplastic tissue. Am J Roentgenol Radium Ther Nucl Med 1972;114:447-59.

25. Hopewell JW, Campling D, Calvo W, Reinhold HS, Wilkinson $J H$, Yeung TK. Vascular irradiation damage: its cellular basis and likely consequences. Br J Cancer Suppl 1986;7:181-91.

26. Rotman M, Seidenberg B, Rubin I, Botstein C, Bosniak M. Aortic arch syndrome secondary to radiation in childhood. Arch Intern Med 1969;124:87-90.

27. Vanetti $E$, Clivio A, Nicolini G, et al. Volumetric modulated arc radiotherapy for carcinomas of the oro-pharynx, hypopharynx and larynx: a treatment planning comparison with fixed field IMRT. Radiother Oncol 2009;92:111-7.

28. Verbakel WF, Cuijpers JP, Hoffmans D, Bieker $M$, Slotman BJ, Senan S. Volumetric intensity-modulated arc therapy vs. conventional IMRT in head-and-neck cancer: a comparative planning and dosimetric study. Int J Radiat Oncol Biol Phys 2009;74:252-9. 